\title{
Simple monoclinic crystal phase in suspensions of hard ellipsoids
}

\author{
P. Pfleiderer* and T. Schilling \\ Institut für Physik, Johannes Gutenberg-Universität, Staudinger Weg 7, D-55099 Mainz, Germany \\ (Received 6 December 2006; revised manuscript received 12 January 2007; published 9 February 2007)
}

\begin{abstract}
We present a computer simulation study on the crystalline phases of hard ellipsoids of revolution. For aspect ratios $\geqslant 3$ the previously suggested stretched-fcc phase [Frenkel and Mulder, Mol. Phys. 55, 1171 (1985)] is replaced by a different crystalline phase. Its unit cell contains two ellipsoids with unequal orientations. The lattice is simple monoclinic. The angle of inclination of the lattice, $\beta$, is a very soft degree of freedom, while the two right angles are stiff. For one particular value of $\beta$, the close-packed version of this crystal is a specimen of the family of superdense packings recently reported [Donev et al., Phys. Rev. Lett. 92, 255506 (2004)]. These results are relevant for studies of nucleation and glassy dynamics of colloidal suspensions of ellipsoids.
\end{abstract}

DOI: 10.1103/PhysRevE.75.020402

PACS number(s): 82.70.Dd, 64.60.Cn, 61.50.Ah, 82.20.Wt

Classical, hard particles such as nonoverlapping spheres, rods, or ellipsoids are widely used as models for granular matter, colloidal and molecular fluids, crystals, and glasses. Their success-and their appeal-lies in the fact that the problem of evaluating a many-body partition function is reduced to a slightly simpler, geometrical problem, namely, the evaluation of entropic contributions only. This is an advantage, in particular, for computer simulations. Hence one of the first applications of computer simulations was a study of the liquid-solid phase transition in hard spheres [1].

In this Rapid Communication, we reexamine the highdensity phase behavior of hard ellipsoids of revolution with short aspect ratios. This system has been studied in Monte Carlo simulations by Frenkel and Mulder in 1985 [2]. Since then, the focus of attention has been on the nematic phase and the isotropic-nematic transition [3-5]. Biaxial hard ellipsoids have also been studied [6,7]. But, to our knowledge, the high-density phases have not been investigated further. Knowledge of these phases is relevant for studies of elongated colloids in general, and it is crucial for the study of nucleation [8] and glassy dynamics [9] in hard ellipsoids.

At high densities, Frenkel and Mulder assumed that the most stable phase was an orientationally ordered solid which can be constructed in the following way. A face-centered cubic (fcc) system of spheres is stretched by a factor $x$ in an arbitrary direction $\mathbf{n}$. This transformation results in a crystal structure of ellipsoids of aspect ratio $x$, which are oriented along $\mathbf{n}$. As the transformation is linear, the density of closest packing is the same as for the closest packing of spheres $\phi$ $=\pi / \sqrt{18} \approx 0.7405$. Recently, Donev and co-workers showed that ellipsoids can be packed more efficiently if non-latticeperiodic packings (i.e. packings in which a unit cell contains several ellipsoids at different orientations) are taken into account [10]. For unit cells containing two particles, they constructed a family of packings which reach a density of $\phi$ $=0.770732$ for aspect ratios larger than $\sqrt{3}$.

We have performed Monte Carlo simulations of hard ellipsoids of revolution with aspect ratios $a / b=\frac{1}{3}, 2,3,4,6$ and found that, for large parts of the high-density phase diagram,

\footnotetext{
*Electronic address: pfleider@uni-mainz.de
}

the lattice crystal suggested by Frenkel and Mulder is unstable with respect to a different crystalline phase. Its unit cell is simple monoclinic and contains two ellipsoids at an angle with respect to each other. We will refer to this phase as SM2 (simple monoclinic with a basis of two ellipsoids).

Simulations were performed at constant particle number $N$, pressure $P$, and temperature $T$. The shape of the periodic box was allowed to fluctuate, so that the crystal unit cell could find its equilibrium shape. This was achieved by implementing the Monte Carlo (MC) equivalent of the simulation method by Parrinello and Rahman [11-13]. We constructed the initial solid structures by stretching a fcc hard sphere crystal along the [111] direction by a factor of $a / b$. Hence the simulations were started with crystals identical to the ones studied by Frenkel and Mulder. For aspect ratios $a / b=\frac{1}{3}, 2,3$, we simulated eight independent systems, each containing $N \approx 1700$ particles. Simulations started at $P$ $=50 k_{\mathrm{B}} T / 8 a b^{2}$. The pressure was lowered in subsequent runs until we observed melting to the nematic phase. In each run, equilibration lasted roughly $2 \times 10^{6} \mathrm{MC}$ sweeps, and was followed by $(1.8-3.2) \times 10^{6} \mathrm{MC}$ sweeps for calculating thermodynamic averages. (One MC sweep consisted of $N$ attempts to move or rotate a particle and one attempt to change the box shape, on average.) For aspect ratio $a / b=6$, we simulated eight independent systems with $N=3072$ at a pressure $P=46 k_{\mathrm{B}} T / 8 a b^{2}$. Equilibration and data acquisition summed up to more than $3 \times 10^{6} \mathrm{MC}$ sweeps. One system with $a / b=4, N=1200$, and $P=46 k_{\mathrm{B}} T / 8 a b^{2}$ was first simulated by sampling only rectangular box shapes for a total of $2.2 \times 10^{6} \mathrm{MC}$ sweeps, and was then simulated including nonrectangular box shapes at the same and lower pressures for several million MC sweeps.

Particle overlap was checked by a routine [14] that uses the Vieillard-Baron [15] and Perram-Wertheim [16,17] criteria. In a simulation of a system with $a / b=3$ and $N=1728$, $1 \times 10^{6} \mathrm{MC}$ sweeps took about $12 \mathrm{~h}$ of CPU time on a $1.8 \mathrm{GHz}$ processor.

All systems with aspect ratios $a / b \geqslant 3$ left the initial fcc structure in favor of a simple monoclinic lattice with a basis of two ellipsoids (SM2). We will discuss this structure for $a / b=3$ and return to different aspect ratios toward the end of this Rapid Communication. 


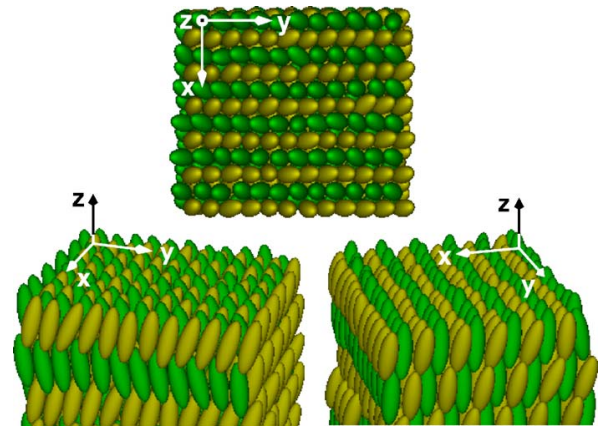

FIG. 1. (Color online) Snapshot of the SM2 crystal from different angles, with $a / b=3, N=1728$, and $P=46 k_{\mathrm{B}} T / 8 a b^{2}$. Color code (grayscale) indicates orientation.

Figure 1 shows a snapshot of a system in the SM2 phase with $a / b=3$. The color code (grayscale) helps distinguish the two directions of orientation which are present in the crystal. In the initial configuration, a stretched-fcc crystal, all ellipsoids were oriented along the $z$ axis. The SM2 phase is mainly the result of a collective reorientation. The two directions of orientation alternate from layer to layer. Layers containing ellipsoids of only one orientation are here parallel to the $y$ axis and form an angle with the $x$ axis. Within layers parallel to the $x y$ plane, the centers of mass of the ellipsoids form a nearly triangular lattice. It differs from the initial fcc crystal by a slight elongation along the $x$ axis. However, the collective reorientation of the ellipsoids displaced their tips in such a way that they now form a rectangular lattice. This can be discerned in the top view in Fig. 1. The tips of the neighboring layers interlace. As a result, each ellipsoid now has four nearest neighbors above and below, whereas in the fcc structure, it had three. The total number of nearest neighbors has increased from 12 to 14 , which is indicative of a higher packing efficiency.

The initial triangular symmetry about the $z$ axis allows for two additional, equivalent SM2 configurations, which are rotated with respect to the one in Fig. 1 by $\pm 60^{\circ}$. We observed these possibilities as well; in fact, only two out of our eight systems assumed the global orientation seen in Fig. 1.

The unit cell of the SM2 structure is shown in Fig. 2. The open circles indicate the centers of the two ellipsoids which form the basis. The cell is monoclinic. The yellow (light gray) ellipsoid is at the origin, the green (dark gray) one is at $\frac{1}{2}(\mathbf{a}+\mathbf{b})$. The orientations are symmetric about the $a c$ plane. The parameters used to produce Figs. 2(a) and 2(b) are thermal average values obtained from simulations with $N$ $=1728$ and $P=46 k_{\mathrm{B}} T / 8 a b^{2}$; cf. Fig. 1 . The cell remained monoclinic even when the pressure was lowered down to the melting transition into the nematic phase.

The angle of inclination, $\beta$, relaxes extremely slowly. The simulations with $N=1728$ were too slow to equilibrate this angle. Therefore we carried out a set of very long simulations for a smaller system $(N=432)$ with initial values of $\beta$ in the range $105^{\circ}<\beta<150^{\circ}$. After more than $100 \times 10^{6}$ Monte Carlo sweeps, there was still no clear evidence for a preferred value of $\beta$. Variations of $15^{\circ}$ in a single simulation were typical, even at $P=46 k_{\mathrm{B}} T / 8 a b^{2}$. Hence, we expect the shear modulus in this degree of freedom to be very small.

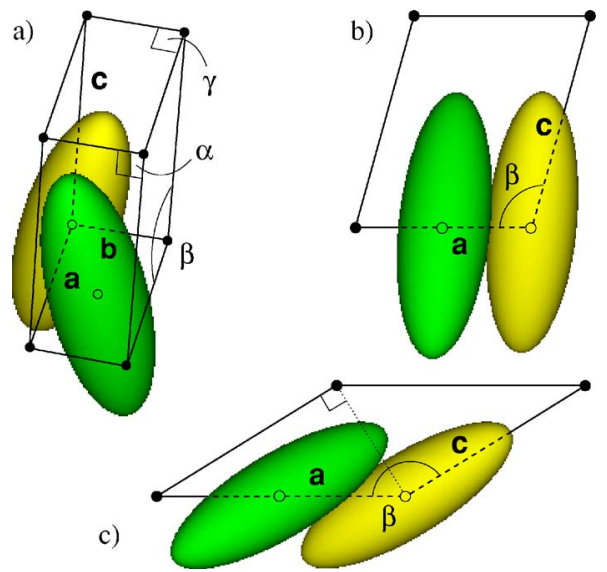

FIG. 2. (Color online) Unit cell of SM2 with $a / b=3$. The open circles indicate the centers of the two ellipsoids which form the basis. The cell is monoclinic. $\beta$ exhibits large variations. The yellow (light gray) ellipsoid is at the origin; the green (dark gray) one is at $(1 / 2)(\mathbf{a}+\mathbf{b})$. The orientations are symmetric about the $a c$ plane. The parameters in (a) and (b) are average values for $N$ $=1728$ and $P=46 k_{\mathrm{B}} T / 8 a b^{2}$; cf. Fig. 1 . (c) shows the cell at close packing with $\beta=148.3^{\circ}$, where it is an instance of the family of packings introduced by Donev et al. [10].

The other two angles $\alpha$ and $\gamma$ were stable at $90^{\circ}$, with fluctuations of less than $1^{\circ}$. The associated shear moduli are much larger. The reason for this interesting rheological property is that planes of uniform orientation slide well past each other in the c direction only. In some of the long simulations, this led to undulations of the lattice in the $\mathbf{c}$ direction to the point of planar defects, which would spontaneously heal again.

To find a lower bound for the maximum density of the SM2 phase $(a / b=3)$, we performed simulations sampling only the unit cell parameters and particle orientations, and imposing all symmetries of SM2. The initial parameters were average values obtained from the simulations with $N=1728$ and $P=46 k_{\mathrm{B}} T / 8 a b^{2}$. In the process of maximizing the density, $\beta$ increased from $105^{\circ}$ to about $150^{\circ}$, and the common tilt of the ellipsoids with respect to the $b c$ plane disappeared. We then imposed that $(\mathbf{a}+\mathbf{c})$ be perpendicular to $\mathbf{c}$ [see Fig. 2 (c)], which is equivalent to $\beta \approx 148^{\circ}$. Under this condition the SM2 structure becomes an instance of the family of packings introduced by Donev et al. [10]. This simulation achieved the highest packing fraction, namely, $\phi$ $\approx 0.770732$ (the value reported by Donev et al.).

But already at $\beta \approx 105^{\circ}$ we found a jamming density of $99.663 \%$ of the maximum. Simulations at intermediate values indicate a smooth approach toward the maximum density as $\beta$ increases. Thus, the close-packing density varies very weakly for $105^{\circ}<\beta<148^{\circ}$. While this range is traversed, ellipsoids of one orientation move past neighbors of the other orientation by almost half their length. This can be seen in Figs. 2(b) and 2(c). For reasons of symmetry, this translation may even continue by the same amount while the density remains above $99.663 \%$ of the maximum. These observations are in accord with the fact that $\beta$ is soft at finite pressures; evidently, the free volume distribution possesses a similarly slight variation with $\beta$. 


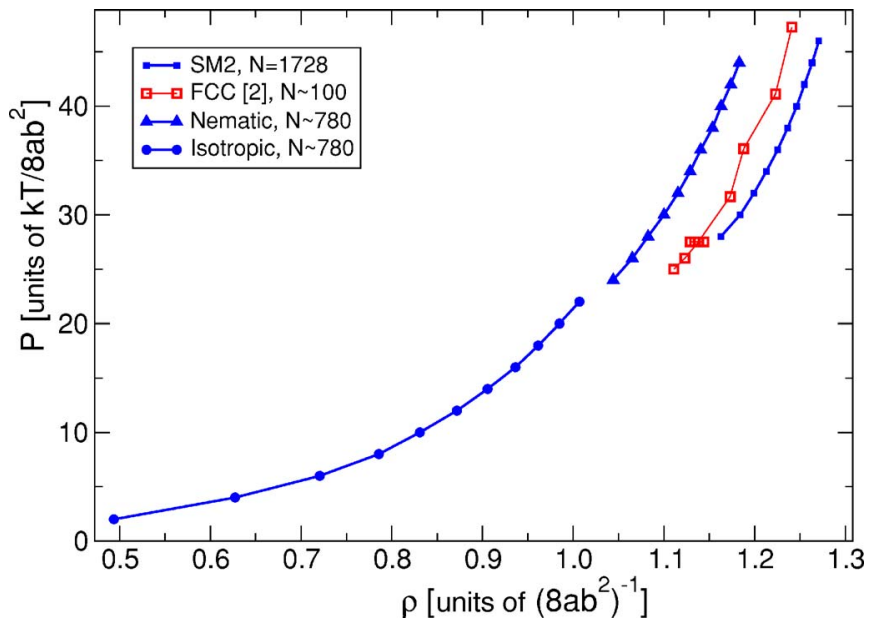

FIG. 3. (Color online) Equation of state data for $a / b=3$ and $N$ $=1728$. The open squares show stretched-fcc data by Frenkel and Mulder [2], the filled squares the higher-density SM2 phase. Also shown are the nematic and fluid branches (triangles and circles, respectively). Errors on our data are indicated by the size of the symbols. The SM2 curve tends to underestimate the density slightly since $\beta$ was not equilibrated entirely.

Figure 3 shows equation of state data of the SM2 phase from our simulations with $N=1728$ particles. The density of the SM2 is higher than that of the stretched-fcc structure for all pressures. Five of eight systems underwent the transition to the SM2 structure already at the highest simulated pressure $P=48 k_{\mathrm{B}} T / 8 a b^{2}$, the remaining three at $P$ $=46 k_{\mathrm{B}} T / 8 a b^{2}$. Note also that in all our runs the SM2 phase melted to the nematic phase without revisiting the stretchedfcc phase from which it developed; evidently, the SM2 not only packs more efficiently than the stretched-fcc phase, it also provides for a better distribution of free volume at all densities until the transition to the nematic phase. Hence it is more stable than the stretched-fcc structure. We also show the nematic branch from an $(N, P, T)$ compression (i.e. the pressure was raised between successive simulations) with $N \approx 780$ particles and up to $6 \times 10^{6} \mathrm{MC}$ sweeps per run. Even at strong overcompression, no spontaneous crystallization occurred. This indicates that the nucleation barrier to the SM2 phase is very high. Also shown is the isotropic fluid branch as obtained from $(N, P, T)$ compression and expansion runs with $N \approx 780$.

All eight simulations at $a / b=6$ and $P=46 k_{\mathrm{B}} T / 8 a b^{2}$ formed the SM2 phase as well, although four of them retained a planar defect. Different regions in the periodic box were able to develop different global orientations of SM2 as the systems were larger $(N=3072)$ than those with $a / b=3$ $(N=1728)$. We also simulated a system with $a / b=4, N$ $=1200$, and $P=46 k_{\mathrm{B}} T / 8 a b^{2}$; it formed the SM2 phase as well. It also developed a planar defect, this time owing to a geometrical mismatch between the simulation box and the SM2 unit cell. Note that for $a / b=3$ and 4 , the SM2 phase formed even in simulations sampling only rectangular box shapes. It is therefore more stable than the stretched-fcc phase even when it cannot assume its equilibrium shape.

By contrast, ellipsoids with $a / b=2$ and the oblate $a / b$

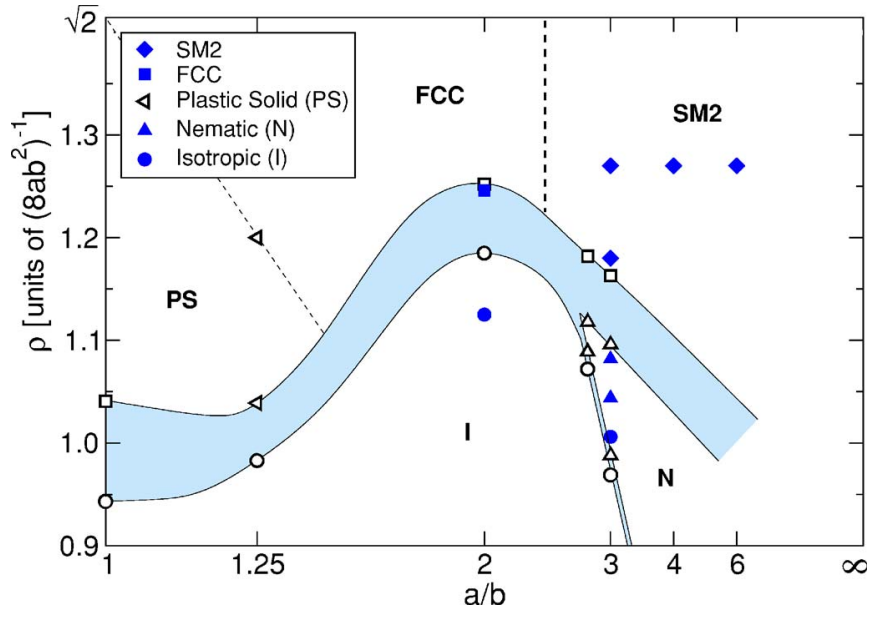

FIG. 4. (Color online) Phase diagram of hard, uniaxial ellipsoids, showing the results of Frenkel and Mulder (open symbols) [2], and their suggested phase boundaries and coexistence regions. The data points at $a / b=1$ are taken from [18]. We have inserted our state points (filled symbols) and a vertical dashed line to delimit the region in which we found the SM2 phase; but our data are insufficient to locate a phase boundary.

$=\frac{1}{3}$ showed no tendency to leave the initial stretched-fcc structure. We studied each of these systems with eight independent simulations. In none of them did two preferred directions of ellipsoid orientation develop. All of them melted to the nematic phase on expansion, directly from the stretched-fcc structure. But note that the apparent stability of fcc in our simulations may well be due to a free energy barrier, rather than indicating genuine stability.

In Fig. 4 we show a phase diagram of hard ellipsoids of revolution. It includes part of the results of Frenkel and Mulder, and their suggested phase boundaries and coexistence regions. We have inserted our state points and a vertical dashed line to delimit the region in which we found the SM2 phase; but our data are insufficient to locate a phase boundary.

In the high-density phase diagram of hard ellipsoids of revolution we have found a crystal that is more stable than the stretched-fcc structure proposed by Frenkel and Mulder [2]. This phase, SM2, has a simple monoclinic unit cell containing a basis of two ellipsoids. The angle of inclination, $\beta$, is a very soft degree of freedom, whereas the other angles are not. At one value of $\beta\left(\approx 148.3^{\circ}\right.$ for $\left.a / b=3\right)$, the closepacked SM2 structure is an instance of the family of packings introduced by Donev et al. [10]. As for thermodynamic stability, our results unequivocally remove the stretched-fcc structure for aspect ratio $a / b=3$ from the phase diagram of hard, uniaxial ellipsoids. Our state points for $a / b=4$ and 6 suggest that this holds for the entire range of $3 \leqslant a / b \leqslant 6$, and possibly beyond. However, this does not prove that SM2 is the ground state. A procedure has been developed for making almost monodisperse ellipsoids $[19,20]$, which are of colloidal size. Their behavior at water-air interfaces has been studied [21,22]; also three-dimensional structural properties of a sedimentation of these particles have been successfully characterized [23]. It would be interesting to perform experiments probing colloidal crystals of ellipsoids. 
This work was supported by the Emmy Noether Program and SFB TR6 of the Deutsche Forschungsgemeinschaft (DFG), and the European Network of Excellence SoftComp.
We are grateful to the NIC Jülich for computing time, and to K. Binder, D. Frenkel, M. P. Allen, J. Vermant, A. Donev, and W. A. Siebel for helpful suggestions.
[1] B. J. Alder and T. E. Wainwright, J. Chem. Phys. 27, 1208 (1957).

[2] D. Frenkel and B. Mulder, Mol. Phys. 55, 1171 (1985).

[3] G. J. Zarragoicoechea, D. Levesque, and J. J. Weis, Mol. Phys. 75, 989 (1992).

[4] M. P. Allen and C. P. Mason, Mol. Phys. 86, 467 (1995).

[5] P. J. Camp, C. P. Mason, M. P. Allen, A. A. Khare, and D. A. Kofke, J. Chem. Phys. 105, 2837 (1996).

[6] M. P. Allen, Liq. Cryst. 8, 499 (1990).

[7] P. J. Camp and M. P. Allen, J. Chem. Phys. 106, 6681 (1997).

[8] T. Schilling and D. Frenkel, Comput. Phys. Commun. 169, 117 (2005).

[9] M. Letz, R. Schilling, and A. Latz, Phys. Rev. E 62, 5173 (2000).

[10] A. Donev, F. H. Stillinger, P. M. Chaikin, and S. Torquato, Phys. Rev. Lett. 92, 255506 (2004).

[11] M. Parrinello and A. Rahman, J. Appl. Phys. 52, 7182 (1981).

[12] R. Najafabadi and S. Yip, Scr. Metall. 17, 1199 (1983).

[13] S. Yashonath and C. N. R. Rao, Mol. Phys. 54, 245 (1985).
[14] M. P. Allen, D. Frenkel, and J. Talbot, Comput. Phys. Rep. 9, 301 (1989).

[15] J. Vieillard-Baron, J. Chem. Phys. 56, 4729 (1972).

[16] J. W. Perram, M. S. Wertheim, J. L. Lebowitz, and G. O. Williams, Chem. Phys. Lett. 105, 277 (1984).

[17] J. W. Perram and M. S. Wertheim, J. Comput. Phys. 58, 409 (1985).

[18] W. G. Hoover and F. H. Ree, J. Chem. Phys. 49, 3609 (1968).

[19] K. M. Keville, E. I. Franses, and J. M. Caruthers, J. Colloid Interface Sci. 144, 103 (1991).

[20] C. C. Ho, A. Keller, J. A. Odell, and R. H. Ottewill, Colloid Polym. Sci. 271, 469 (1993).

[21] M. G. Basavaraj, G. G. Fuller, J. Fransaer, and J. Vermant, Langmuir 22, 6605 (2006).

[22] J. C. Loudet, A. G. Yodh, and B. Pouligny, Phys. Rev. Lett. 97, 018304 (2006).

[23] A. Mohraz and M. J. Solomon, Langmuir 21, 5298 (2005). 